\title{
Ezetimibe Is an Inhibitor of Tumor Angiogenesis
}

\author{
Keith R. Solomon, ${ }^{\star \dagger \ddagger}$ Kristine Pelton, ${ }^{\dagger}$ \\ Kelly Boucher, ${ }^{\dagger}$ Jinsoo Joo, ${ }^{\dagger}$ Christopher Tully, ${ }^{\dagger}$ \\ David Zurakowski, ${ }^{\dagger}$ Carl P. Schaffner, ${ }^{\S}$ \\ Jayoung Kim, ${ }^{\ddagger \llbracket}$ and Michael R. Freeman ${ }^{\ddagger \mp ा \|}$ \\ From the Departments of Orthopaedic Surgery, ${ }^{*}$ Surgery, \\ and Biological Chemistry and Molecular Pharmacology," \\ Harvard Medical School, Boston, Massachusetts; the Departments \\ of Orthopaedic Surgery, ${ }^{\dagger}$ and Urology, ${ }^{\ddagger}$ Children's Hospital \\ Boston, Boston, Massachusetts; and the Department of \\ Microbiology and Biochemistry, ${ }^{\lessgtr}$ Waksman Institute, Rutgers, \\ the State University of New Jersey, New Brunswick, New Jersey
}

Epidemiological and preclinical observations have suggested a role for one or more products of the mevalonate/cholesterol biosynthesis pathway in the progression of prostate cancer. In this study, we used ezetimibe (Zetia), a specific, FDA-approved, cholesterol uptake-blocking drug, in combination with either a hyper- or hypocholesterolemic diet, to show that elevated circulating cholesterol levels promote, whereas a reduction in circulating cholesterol levels retard, the growth of human prostate cancer xenograft tumors in mice. Circulating cholesterol levels also modified tumor angiogenesis; higher cholesterol levels increased microvessel density and other indicators of vascularity. Consistent with these data, the reduction of cholesterol levels also increased the levels of the angiogenesis inhibitor thrombospondin-1 in the xenografts. Our results thus suggest that hypercholesterolemia directly accelerates the growth of prostate carcinomas, and that the pharmacological reduction of serum cholesterol levels may retard prostate cancer growth by inhibiting tumor angiogenesis. (Am J Pathol 2009, 174:1017-1026; DOI: 10.2353/ajpath.2009.080551)

Prostate cancer (PCa) and normal aging prostate tissue accumulate high levels of cholesterol. ${ }^{1-3}$ A role for high levels of serum cholesterol in PCa incidence and progression has been suggested through a number of epidemiological and preclinical studies. ${ }^{1,4-15}$ High fat/high cholesterol 'Western' diets have been linked to PCa incidence and progression in a number of reports, however a role for specific dietary components in disease pro- gression is in dispute. ${ }^{16,17}$ Studies examining groups of nutritional components eaten together suggest that diets with a high content of processed and/or red meat may be associated with higher PCa incidence. ${ }^{18,19}$ In addition, some (but not all) studies of cholesterol-lowering drug use (ie, 3-hydroxy-3-methyl-glutaryl-CoA reductase inhibitors, aka statins) and cancer incidence have shown a negative association between statin use and PCa incidence and/or progression, including a significant reduction in risk of advanced disease with long-term statin use. $^{4,7-10}$ Interestingly, meta-analyses of placebo-controlled studies have not supported a link between statins and $\mathrm{PCa}$ incidence. ${ }^{20-22}$ Although these studies have raised a number of concerns, ${ }^{23-25}$ they do illustrate that aspects of statin dose, duration, type, and mechanism of anti-cancer action likely need to be considered when determining whether statins affect PCa incidence or rates of progression.

Cholesterol is a neutral lipid that plays an important pathological role in cardiovascular disease. Statin drugs have been tested as potential chemotherapeutic agents in a number of experimental contexts, however few studies to date have been designed to assess the role specifically of cholesterol-in isolation from other factors-in cancer growth and progression. Statins interfere with the mevalonic acid/cholesterol synthesis pathway at an early step, thereby blocking formation of isoprenoid intermediates necessary for lipidation of critical signaling proteins, such as the Ras family of guanosine triphosphatases. In cell culture and in animal studies, it is apparent that statins do affect isoprenylation because bypassing isoprenoid synthesis inhibition reverses statin-induced apoptosis. ${ }^{26}$ Effects on isoprenoid synthesis have been proposed as the underlying mechanism for the drugs' anti-tumor effects. ${ }^{27-35}$ However, this mechanism is contentious because statins, at standard doses, may not accumulate in peripheral tissues in a concentration sufficient to interfere with isoprenoid synthesis. ${ }^{36-38}$

Supported by NIH grants R01 CA101046 to K.R.S., R37 DK47556, R01 CA112303 to M.R.F., and US Army DoD grant PC050337 to M.R.F., and the Japan Foundation for Aging and Health.

Accepted for publication November 12, 2008.

Address reprint requests to Keith R. Solomon, Ph.D., Dept. of Orthopaedic Surgery, Children's Hospital Boston, Enders 1030, 300 Longwood Avenue, Boston, MA 02115. E-mail: keith.solomon@childrens.harvard.edu 
In a previous study we demonstrated that the atherogenic Paigen diet, which causes hypercholesterolemia, stimulates growth of LNCaP human PCa xenografts. ${ }^{12}$ Tumors in the hypercholesterolemic environment accumulated cholesterol in lipid raft membranes, exhibited lower levels of apoptosis, and enhanced activation of Akt, a serine-threonine kinase linked to aggressive cancers. In explaining these results, we proposed that cholesterol may be directly contributing to tumor growth by altering signal transduction through effects on lipid rafts. ${ }^{1,12,39}$

In the present study we isolated cholesterol from other potential factors, such as isoprenoids, using ezetimibe, a cholesterol-lowering drug that binds to and blocks NPC1L1, the gut transporter responsible for dietary and biliary cholesterol absorption. ${ }^{40-44}$ Our findings, using this drug in combination with other approaches, support a direct role for circulating cholesterol in the promotion of tumor growth and further suggest that an underlying mechanism of this effect occurs at the level of tumor angiogenesis.

\section{Materials and Methods}

\section{Antibodies}

The following antibodies were obtained from the sources indicated: anti-CD31 mAb (rat anti-mouse) and anti-caveolin pAb (BD Pharmingen, San Jose, CA); anti-thrombospondin-1 pAb, anti-caveolin-1 pAb, and anti-Ki-67 pAb (Abcam, Cambridge, MA); anti- $\beta$ actin mAb and anti-smooth muscle actin mAb (Sigma, St Louis, $\mathrm{MO}$ ); anti-phosphotyrosine mAb (Cell Signaling, Danvers, MA); anti-fibroblast mAb (Santa Cruz Biotechnology, Santa Cruz, CA); Alexa Fluor 488-conjugated goat antirat, Alexa Fluor 488-conjugated goat anti-mouse, Alexa Fluor 568-conjugated goat anti-rabbit, Alexa Fluor 568conjugated goat anti-mouse (Invitrogen, Carlsbad, CA); and Cy3-conjugated AffiniPure goat anti-rabbit IgG, Fc fragment specific (Jackson ImmunoResearch, West Grove, PA).

\section{Mice and Tumor Xenografts}

Five-week-old SCID mice were obtained from the Massachusetts General Hospital and were fed a low fat/no cholesterol diet (LFNC) (Research Diets, New Brunswick, NJ diet \# D12102) for 2 weeks, blood was drawn from the saphenous tail vein, and the serum cholesterol concentration was determined using the Infinity Cholesterol Liquid Stable Reagent (Thermo Electron Corp., Waltham, MA). The mice were then divided into high fat/high cholesterol diet (HFHC) (Research Diets, diet \# D12108) and LFNC diet groups with and without ezetimibe $(30 \mathrm{mg} / \mathrm{kg} /$ day; Schering-Plough, New Brunswick, NJ, added to powdered food) and the mice continued on these diets for 2 weeks before tumor implantation. Xenografts were initiated by injecting LNCaP $\left(2 \times 10^{6}\right.$ per site) with 1:1 volume of Matrigel (BD BioSciences, San Jose, CA) into the four dorsal quadrants of each animal. To eliminate any injection bias, the mice were randomized before implantation and the implanter was blinded to which group each mouse was assigned. All animal procedures were done in compliance with Children's Hospital Boston's animal care and use policies. Tumors were measured daily from the initiation of the first palpable tumors and the mice were sacrificed before reaching the maximum tumor burden ( $\approx 13$ days post implantation). Terminal bleeds were taken (cardiac puncture) for serology (triglyceride, bilirubin and other liver function tests were performed in the Dept. of Laboratory Medicine, Children's Hospital Boston; androgen levels were determined by a testosterone EIA, Diagnostic Systems Laboratories, Webster, TX). Tumors were removed, measured, weighed, and either
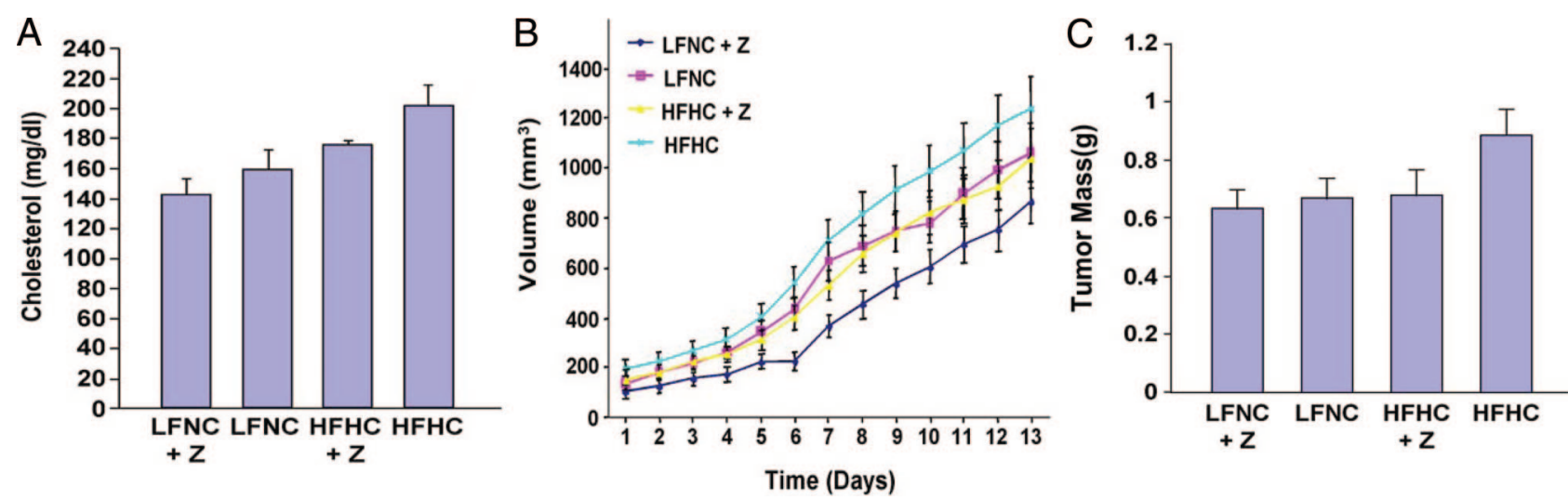

Figure 1. Diet/ezetimibe effects on serum cholesterol levels and the growth of implanted prostate tumors in SCID mice. A: Serum cholesterol levels in diet/ezetimibe mouse cohorts. Animals were given various ezetimibe (Z)-diet combinations (see Figure) for 2 weeks after which the mice were bled by small tail vein incision, and cholesterol measured in the collected serum via Infinity colormetric assay. Data are plotted as cholesterol level (mg/dL) vs. group \pm SE. $n=$ 13 to 15 /group. Two-way analysis of variance indicated significant effects of diet $(\mathrm{F}=77.57, P<0.001)$ and drug (F $=23.48, P<0.001)$ on cholesterol levels, but no significant diet-by-drug interaction $(\mathrm{F}=0.86, P=0.36)$ suggesting that the effects are independent. B: Longitudinal volume measurements. SCID mice were fed various diet/ezetimibe (Z) combinations for 2 weeks before tumor implantation (see Materials and Methods). Tumors were measured daily by calipers starting at first appearance (day 1$)$ and continued for 13 days. Data are plotted as tumor volume $\left(\mathrm{mm}^{3}\right)$ per site versus time (days) \pm SE. A mixed model analysis was used to calculate the significance of the both diet $(P=0.048)$ and ezetimibe $(\mathrm{Z})(P=0.035)$ on tumor growth. $n=52$ to $60 /$ group. C: Tumor wet weight. At sacrifice all tumors were removed and weighed. Data are plotted as average tumor mass $(\mathrm{g})$ per site versus group \pm SE. These data were statistically significan between LFNC $+Z(0.63 \pm 0.55$ average grams/tumor site $)$ vs. HFHC $(0.88 \pm 0.71$ average grams/tumor site $)$ groups $(P=0.021)$ and between the LFNC $(0.67 \pm$ 0.56 average grams/tumor site) vs. HFHC groups $(P=0.037) . n=52$ to $60 /$ group. In all cases data are considered significant at $P<0.05$. 


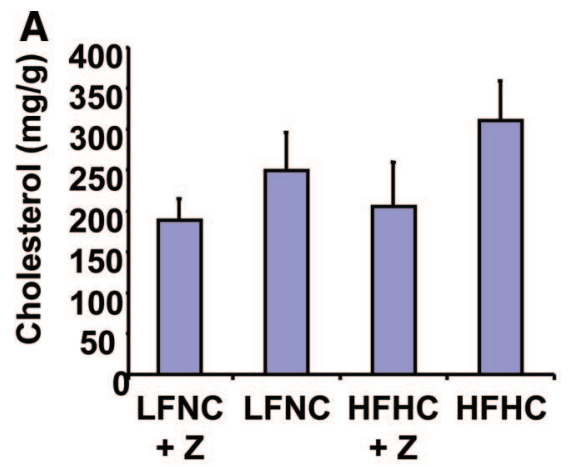

B
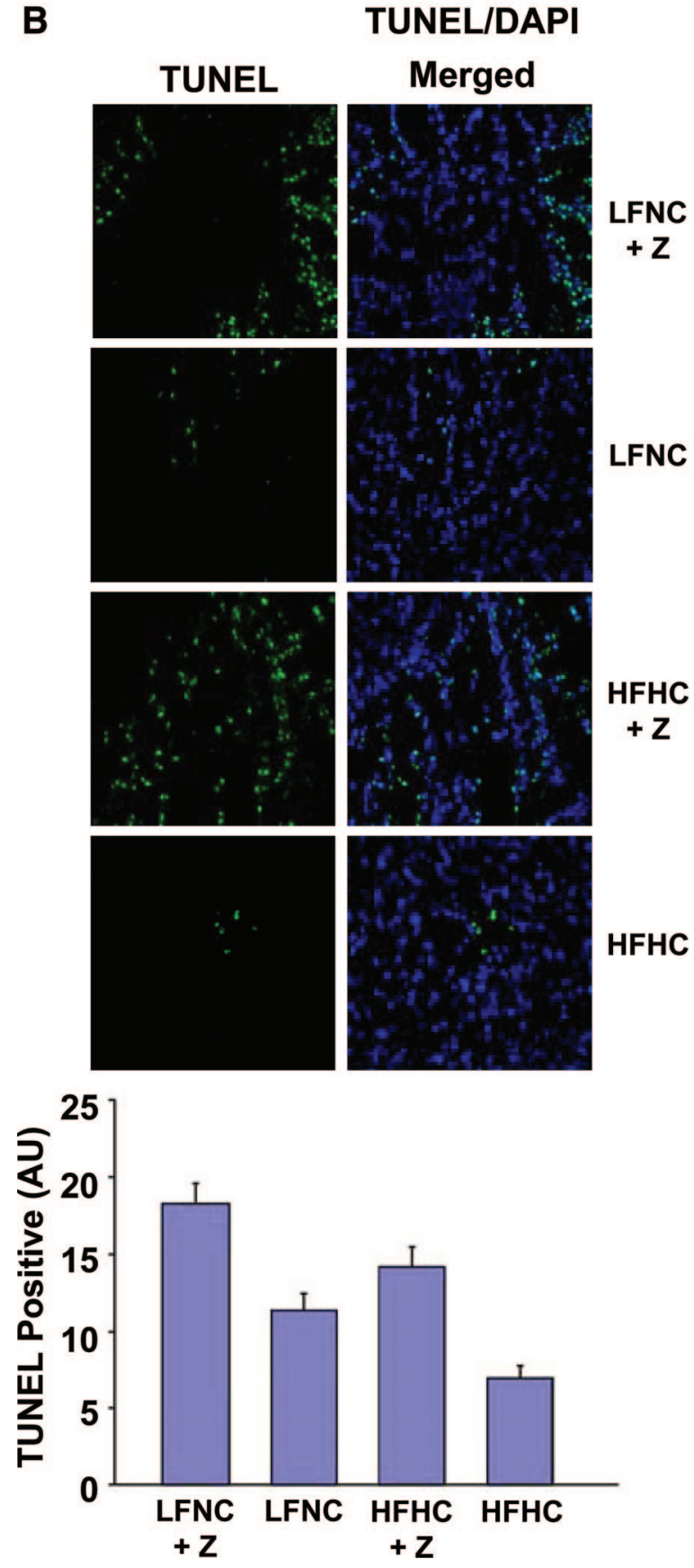

Figure 2. Biochemical and cell biological tumor characteristics. A: Tumor cholesterol levels. Membranes were prepared from tumors and subjected to cholesterol extraction and analysis (see Materials and Methods). Data are plotted as cholesterol (mg)/g tumor tissue versus diet/ezetimibe (Z) group \pm SE. Data were analyzed by analysis of variance, which indicated no significant interaction between diet and ezetimibe $(P=0.40)$ but highly significant main effects of both diet $(P=0.039)$ and ezetimibe $(P<0.0001) n=9$ /group. B: Tumor cell apoptosis (TUNEL staining). Upper panel: representative TUNEL stained images. Left column shows TUNEL staining (fluorescein; green) of selected tumor sections; right column is the merged image of TUNEL and DAPI counterstaining. $n=$ 40. Lower panel: quantitative evaluation of tumor cell apoptosis levels. Data are plotted as relative level of TUNEL staining versus diet/ezetimibe $(\mathrm{Z})$ group \pm SE. Data were analyzed by analysis of variance, which indicated no significant interaction between diet and ezetimibe $(P=0.85)$ but highly significant main effects of both diet $(P<$ $0.0001)$ and ezetimibe $(P<0.0001)$. C: Tumor cell proliferation (Ki-67 staining). Upper panel: representative Ki-67 stained images. Left column, Ki-67 staining (red); Right column, merged image of Ki-67 (Cy3; red) and DAPI (blue; nuclei) counterstaining. $n=20$. Lower panel: quantitative evaluation of tumor cell proliferation levels. Data are plotted as relative level of Ki-67 staining versus diet/ezetimibe ( $\mathrm{Z}$ ) group $\pm \mathrm{SE}$. The data were analyzed by two-way analysis of variance, which demonstrated that there is a significant ezetimibe by diet interaction $(P=$ 0.027), implying that ezetimibe has a significant effect on lowering proliferation although the magnitude of this effect depends on the diet; LFNC: average 28.78 (95\% CI; 25.42 to 32.14) w/ezetimibe: average 25.57 (95\% CI; 22.21 to 28.93); HFHC: average 41.16 (CI; 37.80 to 44.52$)$ w/ezetimibe: average 30.32 (95\% CI; 26.96 to 33.68 ). Images were acquired and analyzed by AxioVision 4.0 software for quantification. In all cases data are considered significant at $P<0.05$.
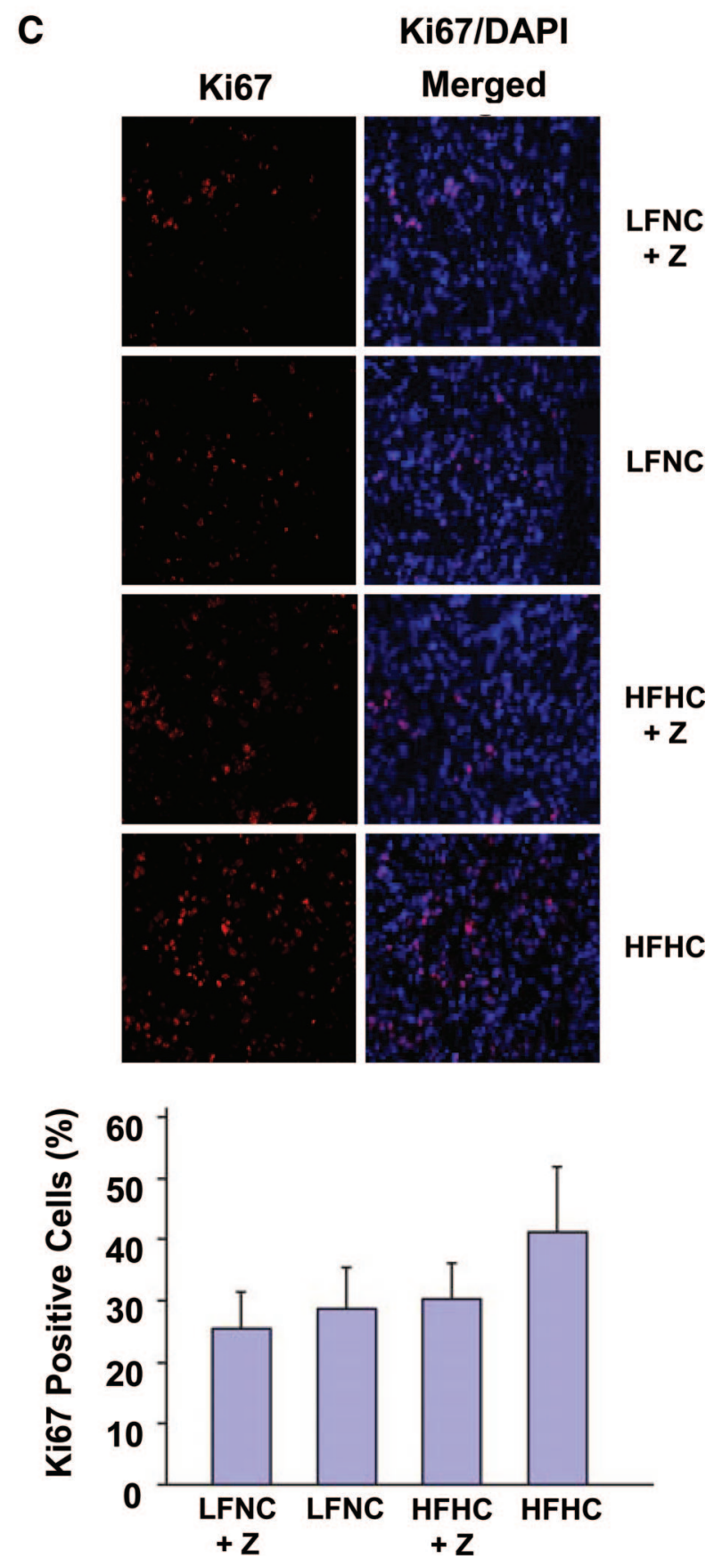

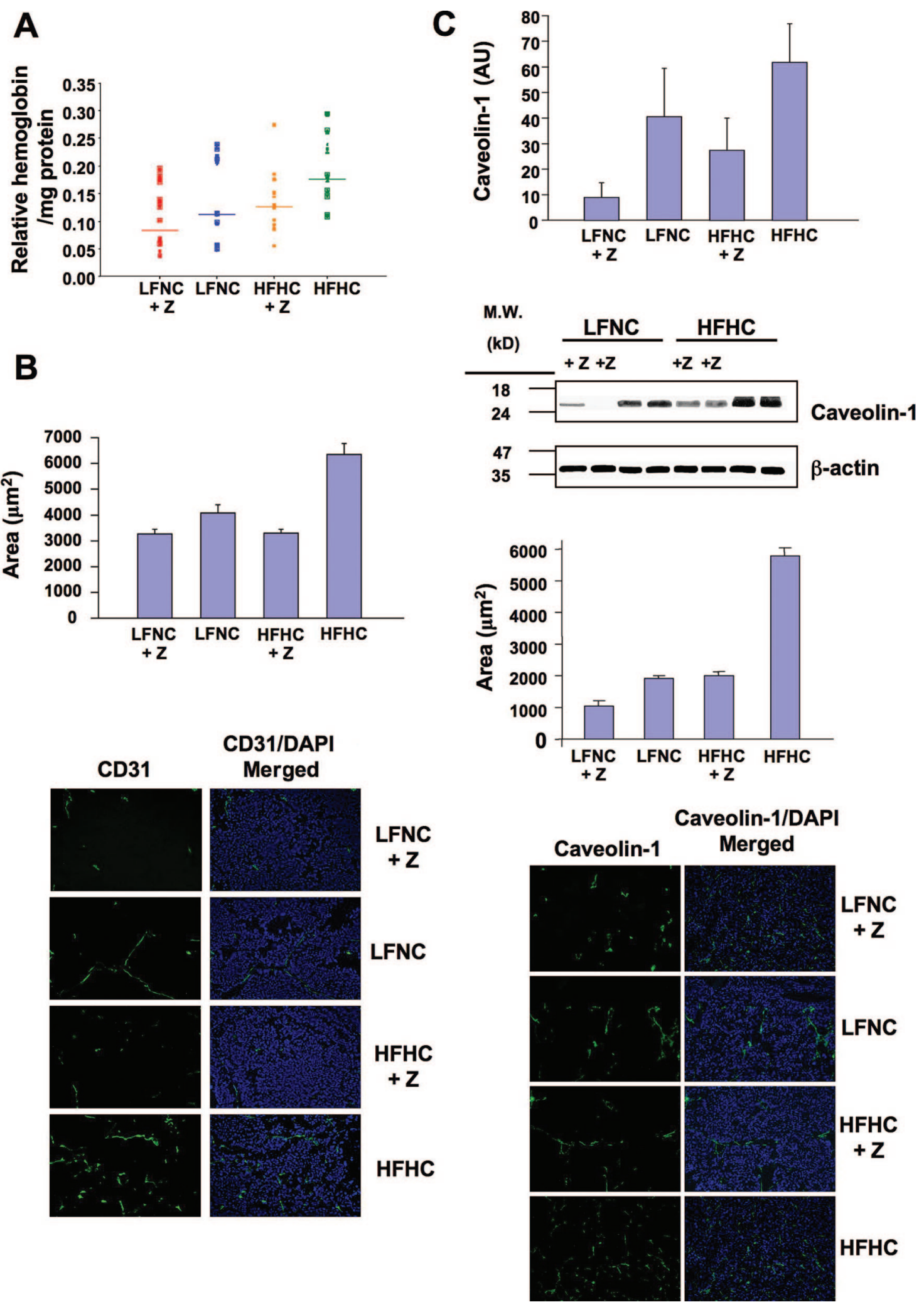
placed in optimal cutting temperature (OCT) solution (Tissue-Tek, Torrance, CA) or snap frozen.

\section{Cell Culture}

LNCaP human prostate tumor cells (American Type Culture Collection, Manassas, VA), which do not express either caveolin ${ }^{39}$ or phosphatase and tensin homolog ${ }^{45}$ were cultured in RPMI (Invitrogen, Carlsbad, CA) media supplemented with $10 \%$ fetal bovine serum and $1 \%$ penicillin/streptomycin at $5 \% \mathrm{CO}_{2}$ at $37^{\circ} \mathrm{C}$.

\section{Tumor Cholesterol Analysis}

Tumors were finely minced in PBS on ice and the level of cholesterol was determined as described previously. ${ }^{26}$

\section{Apoptosis}

Percentage of apoptotic cells in tumor sections was determined by terminal deoxynucleotidyl transferase dUTP nick-end labeling (TUNEL) assay using the In Situ Cell Death Detection Kit (Roche Diagnostics Corp. Indianapolis, IN). Briefly, frozen tumor sections were fixed in $4 \%$ paraformaldehyde, permeabilized and the DNA stained with fluorescein following the manufacturer's instructions. Nuclei were counterstained with 4,6-diamidino-2-phenylindole (DAPI, Vector Labs, Burlingame, CA). Images were captured using a Zeiss microscope and the positive cells and nuclei were counted using Axiovision software 4.0 .

\section{Immunofluorescence}

Tumor samples frozen in OCT were sectioned (at 3 to 20 $\mu \mathrm{m}$ ), mounted on Superfrost slides (ThermoFisher Scientific, Waltham, MA), and air-dried for 30 minutes. Sections were then fixed using cold acetone (5 minutes), followed by $1: 1$ acetone:chloroform ( 5 minutes), and then acetone (5 minutes) or by using $4 \%$ paraformaldehyde at room temp (30 minutes) followed by $0.025 \%$ PBS/Triton X-100 (5 minutes) to permeabilize the cells. Sections were washed with cold PBS 3 times for 5 minutes each and were incubated in a protein blocking solution of PBS/
Tween (0.1\%) with $5 \%$ bovine serum albumin (Sigma) at room temp (30 minutes), and were then washed in cold PBS 3 times for 5 minutes each. The appropriate primary antibodies, diluted 1:200 to 1:1000 in blocking solution, were incubated with the sections overnight at $4^{\circ} \mathrm{C}$. Sections were washed 3 times for 5 minutes in cold PBS, and incubated in blocking solution (10 minutes). The sections were then incubated with the appropriate fluorescent secondary reporter antibodies diluted 1:500 to 1:5000 in blocking solution at room temp (30 minutes) followed by washing 3 times in PBS. Nuclei were counterstained with DAPI. Double staining of CD31 with thombospndin-1 (TSP-1) and CD31 with smooth muscle actin (SMA) were performed sequentially as described above. Blood vessel and fibroblast analyses were done using IPLab software (BD Biosciences Bioimaging, Rockville, MD) as previously described. ${ }^{46} \mathrm{TSP}-1$ analysis was done using the outline spline in AxioVision 4.0 software. Ki-67 positive cells and nuclei were quantified using AxioVision 4.0 software.

\section{Lysates and Immunoblotting}

Tumors were finely minced in PBS on ice and lipid raft and non-raft fractions were prepared as previously described. ${ }^{47,48}$ Protein concentrations were determined by microBCA (Pierce/Thermo Scientific) and equal amounts of the lysates were subjected to SDS-polyacrylamide gel electrophoresis and immunoblotting as described previously. ${ }^{12}$

\section{Hemoglobin Assay}

Tumors were finely minced in $1 \mathrm{ml}$ of fresh PBS on ice. The minced tissue suspensions were centrifuged at $10,000 \times g$ at $4^{\circ} \mathrm{C}$ (2 minutes) and the supernatant removed. The optical density of the clarified supernatants was read at $650 \mathrm{~nm}$ (background) and $530 \mathrm{~nm}$ (hemoglobin) using a spectrophotometer. ${ }^{49,50}$

\section{Statistics}

To assess the effects of drug and diet on growth as well as microvessel density, pericyte coverage, and TSP-1

\footnotetext{
Figure 3. Angiogenesis in xenograft tumors. A: Tumor hemoglobin quantification. Tumors were subjected to mechanical disruption in PBS, followed by centrifugation (to remove debris) and the clarified supernatants were analyzed by optical density (absorbance at $530 \AA$ - absorbance at $650 \AA$ ). Data are plotted as relative hemoglobin/mg tumor tissue versus group (mean value is indicated by line). All groups demonstrated statistical significance versus all other groups except for LFNC versus HFHC + Z (ezetimibe), and LFNC versus LFNC + Z, which were not statistically different. $n=20$. B: MVD-CD31 analysis. Upper panel quantitative evaluation of tumor section CD31 levels. Data are plotted as relative level of CD31 staining versus diet/ezetimibe (Z) group \pm SE. Data were analyzed by mixed model analysis, which indicated no significant interaction between diet and ezetimibe $(P=0.199)$ but highly significant main effect of ezetimibe $(P=$ $0.013)$ with larger effects when the HFHC was used $(P=0.01)$. Lower panel: representative anti-CD31 mAb stained images. Left column, CD31 staining (Alexa Fluor 488; Green); Right column, merged image of CD31 (Alexa Fluor 488; Green) and DAPI (blue; nuclei) counterstaining. $n=76$ to 103 . C: MVD-caveolin-1 analysis: Immunoblotting. Tumors were subjected to SDEM and raft fractions were then subjected to SDS-polyacrylamide gel electrophoresis and immunoblot analysis using anti-caveolin-1 mAb. Film exposures were analyzed by densitometry and the signal intensity data normalized for each film so that the largest signal had a value of 1 . All signal ratios were then averaged to calculate the relative intensity of caveolin-1 for tumor samples from each diet/drug cohort. Data are plotted as average caveolin-1 signal (arbitrary units) vs. diet/ezetimibe (Z) group \pm SE. Analysis of variance analysis indicated no statistically significant diet effect $(P=$ 0.171 ) however a significant drug effect in lowering caveolin for both diet conditions $(P=0.027)$. $n=6 /$ group. Immunofluorescence. Upper panel Quantification of caveolin-1 staining. Data are plotted as relative level of caveolin-1 staining versus diet/ezetimibe (Z) group \pm SE Data were analyzed by mixed model analysis, which indicated highly significant main effects of ezetimibe $(P<0.0001)$ and diet $(P<0.0001) . n=43$ /group. Lower panel; representative caveolin-1 staining. Sections were stained for caveolin-1 (see Materials and Methods) and nuclei were counterstained with DAPI. Left column; representative anti-caveolin-1 mAb staining (Alexa Fluor 488; green). Right column; representative caveolin-1 staining (Alexa Fluor 488; green) merged with DAPI staining (blue). Tumors were fixed in OCT, and $10 \mu \mathrm{m}$ sections were stained for the indicated markers. CD31 (PECAM) staining was performed as described. ${ }^{46}$ Fluorescent mages were acquired and analyzed by AxioVision 4.0 software for quantification. In all cases data are considered significant at $P<0.05$.
} 
levels in xenograft tumors in SCID mice, a mixed-model analysis of variance was used to account for the repeated measurements within the same tumor or animal and multiple time points. ${ }^{51}$ Compound symmetry covariance
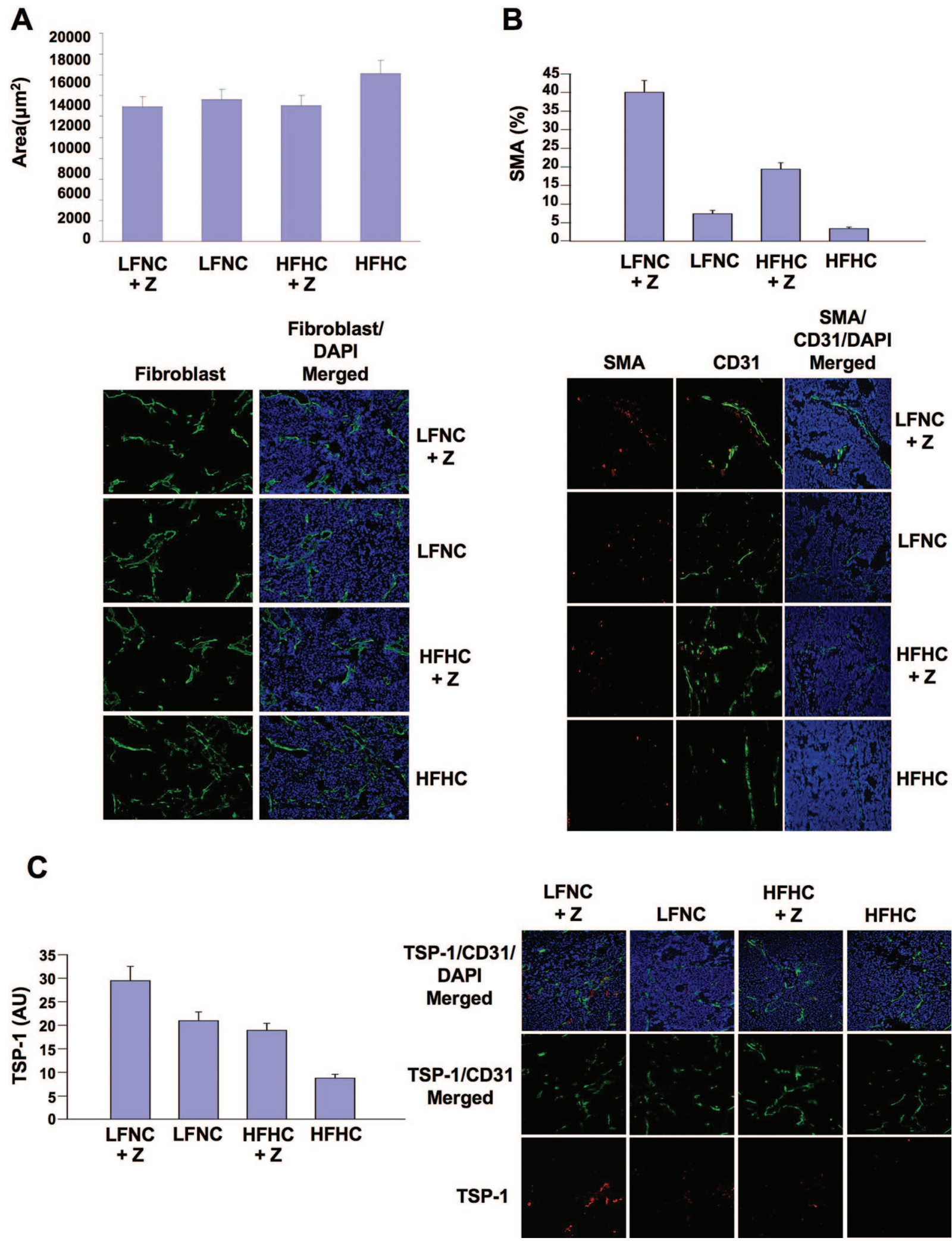

structure was incorporated to model the within-animal correlation and provided the best fit to the data as judged by Akaike's information criterion. ${ }^{52}$ Data for tumor cholesterol, apoptosis, and proliferation were analyzed using

B
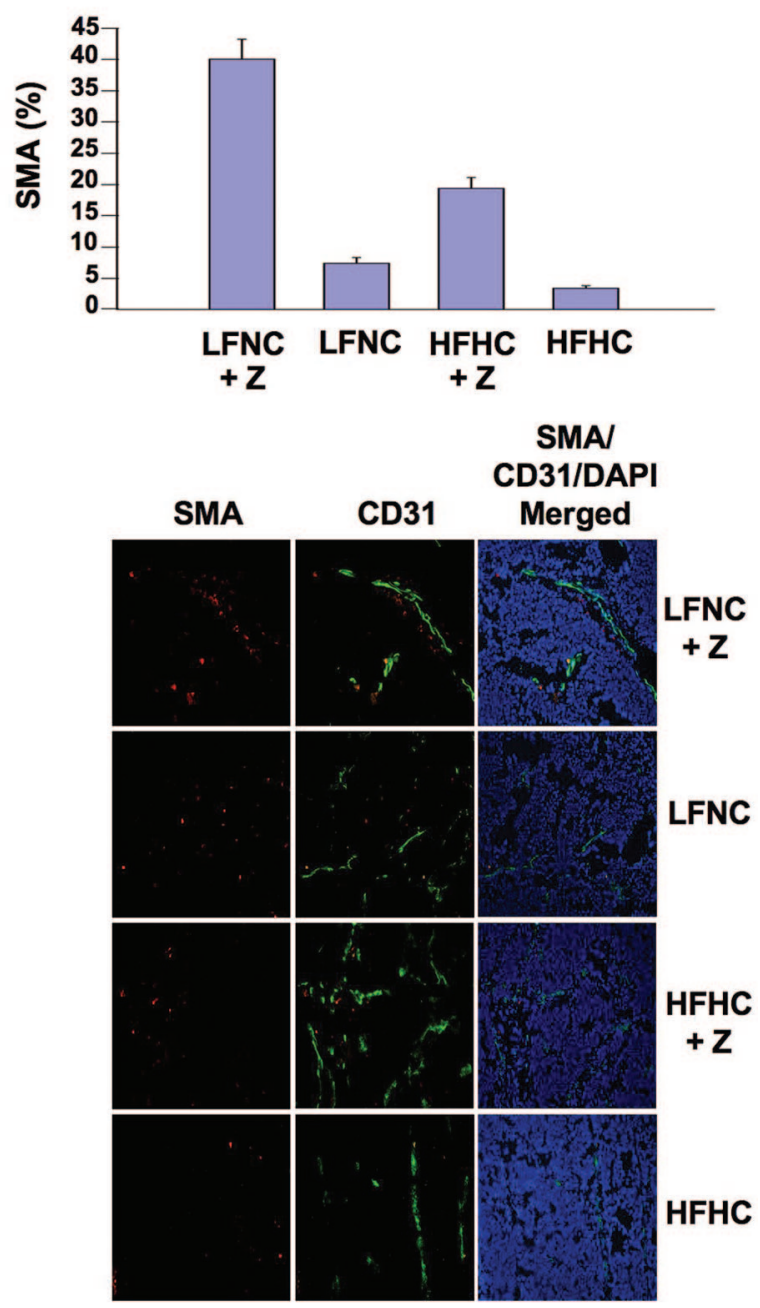
two-way analysis of variance with diet and drug as factors in the $2 \times 2$ factorial experiment and diet-bydrug interaction term in the models to ascertain whether the diet and drug effects are independent main effects or conditional. ${ }^{53}$ Simple comparisons were performed using the standard unpaired Student's t-test. Statistical analysis was performed with SPSS software (version 15.0, SPSS Inc., Chicago, IL). Twotailed values of $P<0.05$ were considered statistically significant.

\section{Results}

The atherogenic Paigen diet is the standard method for raising levels of circulating cholesterol in mice. ${ }^{54}$ The Paigen diet causes severe liver toxicity ${ }^{55}$ and contains sodium cholate, a bile acid, and liver toxin. To isolate the effect of cholesterol from other factors under conditions more relevant to human diets, we attempted to raise cholesterol using a HFHC diet without sodium cholate. Application of this diet resulted in significantly elevated serum cholesterol levels (Figure 1A), thus allowing the comparison of mice made hypercholesterolemic by this diet to animals fed a normocholesterolemic LFNC diet. These diets are isocaloric, excluding any possibility of an energy effect. A subset of mice on both diets was treated with ezetimibe (30 mg/kg/d) to lower cholesterol.

Initially, cholesterol levels were normalized in all animals using the LFNC diet for 2 weeks. Average cholesterol level was $147.47 \pm 17.25 \mathrm{mg} / \mathrm{dL}$ (range 112.07 to $214.66 \mathrm{mg} / \mathrm{dL}$ ). Note that standard mouse chow contains only $0.002 \%(\mathrm{w} / \mathrm{w})$ cholesterol and this level of cholesterol in the diet does not alter murine serum cholesterol levels versus a diet containing no cholesterol (data not shown). Two mice with the lowest and highest cholesterol levels were eliminated from the study because cholesterol varied by more than $2 S D$ from the average. Fifty-eight mice were then randomly assigned to 1 of 4 diet/drug groups : (1) LFNC, (2) LFNC+ezetimibe (Z), (3) HFHC, and (4) $\mathrm{HFHC}+\mathrm{Z}$. Calorie intake was set at $21.18 \mathrm{Kcal} / \mathrm{d}$ in all groups. Observation indicated that ezetimibe did not alter feeding behavior. Animals were kept on the regimens for 2 weeks, and cholesterol levels determined, at which point serum cholesterol levels were significantly different between the four cohorts (Figure 1A). Ezetimibe caused significant reductions in serum cholesterol in both the LFNC and HFHC diet groups.

Following alteration of cholesterol levels, the animals were injected subcutaneously with $2 \times 10^{6} \mathrm{LNCaP}$ cells/ site in their flanks (four tumors/mouse). Regimens were continued following implantation, and the experiment continued for 13 days following the initial appearance of tumors. Tumor volume was measured daily (Figure 1B). Significantly reduced tumor growth rates were observed with the LFNC diet $(P=0.048)$ and by added ezetimibe $(P=0.035)$. The overall effect of diet and ezetimibe was independent and additive, not synergistic. HFHC tumor wet weights at sacrifice were significantly larger than tumors in the other groups (Figure 1C; LFNC drug $(0.63 \pm 0.55 \mathrm{~g})$ vs. HFHC $(0.88 \pm 0.71 \mathrm{~g}), P=0.021$ LFNC $(0.67 \pm 0.56 \mathrm{~g})$ vs. HFHC, $P=0.037)$. Tumor take was $>95 \%$ in all groups and no significant differences in tumor take between groups were observed. We expected that long-term maintenance on the HFHC diet might raise serum triglycerides; conversely, ezetimibe has been reported to exert a modest reduction of triglyceride levels in humans. ${ }^{41,42}$ However, in the present study, the HFHC diet did not result in statistically significant increases in serum triglycerides, nor were triglyceride levels significantly reduced in the ezetimibe cohorts (data not shown). Serological testing (AST, ALP, bilirubin, etc) indicated no liver dysfunction in any mouse (data not shown).

To investigate the mechanisms behind the apparent tumor-promoting effect of elevated cholesterol, we examined the tumors using a variety of approaches. The level of membrane cholesterol in the tumors reflected the serum cholesterol levels in vivo (Figure 2A). Tumor cholesterol levels were independently affected by both ezetimibe and by diet. Apoptosis and cell proliferation were evaluated quantitatively in the tumors. Apoptosis was significantly increased by the LFNC diet $(P<$ $0.0001)$ and independently by ezetimibe $(P<0.0001)$, however, no synergy was observed $(P=0.85)$ (Figure $2 \mathrm{~B}$ ). Cell proliferation, as measured by $\mathrm{Ki}-67$ staining, was greater in the HFHC tumors and, independently, when ezetimibe was omitted (Figure 2C). A significant diet/ezetimibe interaction ( $P=0.027$ ) was observed using cell proliferation as an endpoint.

In processing the xenografts, we noted that tumors from the HFHC mice were bloodier than tumors from

\footnotetext{
Figure 4. Characterization of the xenograft tumor microenvironment. A: Fibroblast analysis. Upper panel; Quantification of fibroblast staining. Data are plotted as relative level of fibroblast staining versus diet/ezetimibe $(Z)$ group \pm SE. Data were analyzed by two-way analysis of variance analysis, which indicated no statistically significant diet effect, however a statistically significant ezetimibe effect in the HFHC condition was detected $(P=0.03) . n=55 /$ group. Lower panel; representative fibroblast staining. Sections were stained using a fibroblast specific mAb (see Materials and Methods) and nuclei were counterstained with DAPI Left column; representative anti-fibroblast mAb staining (Alexa Fluor 488; green). Right column; representative fibroblast staining (Alexa Fluor 488; green) merged with DAPI staining (blue). B: Pericyte coverage (vessel quality). Pericyte coverage of microvessels was determined by SMA staining of peri-microvesicular regions of stained tumor sections (CD31 staining). Upper panel: quantitative evaluation of tumor section SMA levels. Data are plotted as relative level of SMA staining versus diet/ezetimibe $(Z)$ group \pm SE. Data were analyzed by mixed model analysis, which indicated no significant interaction between diet and ezetimibe (Z) $(P=0.062)$ but highly significant main effects of ezetimibe $(P<0.0001)$ and diet $(P=0.009)$. Lower panel: representative anti-SMA/anti-CD31 mAb stained images. Columns left to right: SMA staining (Alexa Fluor 568; Red); SMA (Alexa Fluor 568; Red) + CD31 (Alexa Fluor 488; Green) merged images; DAPI (blue), SMA (Alexa Fluor 568; Red), + CD31 (Alexa Fluor 488; Green) merged images. $n=34$ to 36. C: TSP-1 levels in tumors. TSP-1 levels in tumor sections were determined by staining tumor sections with anti-TSP-1 and anti-CD31 mAbs. Left panel: quantitative evaluation of tumor section TSP-1 levels. Data are plotted as relative level of TSP-1 staining versus diet/ezetimibe $(\mathrm{Z})$ group \pm SE. Data were analyzed by mixed model analysis, which indicated highly significant main effects of ezetimibe $(P<0.0001)$ and diet $(P<0.0001) . n=34-39$. Right panel; representative anti-TSP-1 (Alexa Fluor 568)/anti-CD31 (Alexa Fluor 488) mAb stained images. Columns top to bottom: DAPI (blue), TSP-1 (Alexa Fluor 568; Red), + CD31 (Alexa Fluor 488; Green) merged images; TSP-1 (Alexa Fluor 568; Red) + CD31 (Alexa Fluor 488; Green) merged images; TSP-1 staining (Alexa Fluor 568; Red). Tumors were fixed in OCT, and either $10 \mu \mathrm{m}$ sections (part A) or $20 \mu \mathrm{m}$ sections (parts $\mathbf{B}+\mathbf{C}$ ) were stained for the indicated markers. CD31 (PECAM) staining was performed as described. ${ }^{46}$ Fluorescent mages were acquired and analyzed by AxioVision 4.0 software for quantification. In all cases data are considered significant at $P<0.05$.
} 
other cohorts. Relative hemoglobin levels correlated positively with circulating cholesterol level (Figure 3A), suggesting a potential effect of cholesterol on vascular penetration into the tumors. Microvessel density (MVD) was quantified using an antibody for CD31 (PECAM), an endothelial cell marker. MVD was significantly suppressed by both ezetimibe and by the LFNC diet when compared with the HFHC diet (Figure 3B). A similar result was observed when caveolin-1, which is expressed at high levels by murine endothelial cells but not by LNCaP cells, was used as an independent assessment of MVD ${ }^{39,56,57}$ (Figure 3C). MVD was not simply a reflection of tumor size because no correlation was observed between tumor size and MVD when similar-sized tumors from each cohort were compared ( $r=0.05 ; P=0.39$ ).

The increase in endothelial cells in tumors grown under hypercholesterolemic conditions suggested the possibility that other stromal cell components may also be affected by the increase in cholesterol level. To assess what contribution fibroblasts made to the tumor we stained tumor sections using a fibroblast specific monoclonal antibody (Figure 4A). This analysis revealed a statistically significant increase in the number of fibroblasts in tumors grown under hypercholesterolemic conditions. Ezetimibe treatment significantly reduced the number of fibroblasts found in the tumors, but only when the HFHC diet was used.

Blood vessels undergoing rapid angiogenesis in tumors tend to exhibit poor vascular morphology, characterized by low pericyte recruitment. ${ }^{58}$ Pericyte coverage of vessels was dramatically increased in the ezetimibe groups (Figure 4B), suggesting a stabilizing effect of lower levels of circulating cholesterol on blood vessel structure. These results strongly suggest that one or more angiogenic factor(s) might be responsible for the observed effects, but after extensive testing we could not find any differences in the cohorts in the pro-angiogenic factors VEGF or bFGF expression (data not shown). Because in our prior studies we had shown that hypercholesterolemia leads to increased activation of the serinethreonine kinase Akt, ${ }^{12}$ and Akt is known to reduce expression of TSP-1, a potent angiogenic suppressor, ${ }^{59}$ we examined TSP-1 levels in the tumors. Here we found highly significant differences in TSP-1 levels, which were enhanced by both the LFNC diet and ezetimibe (Figure 4C). Collectively, these results indicate a pronounced effect of circulating cholesterol on mechanisms of angiogenesis.

\section{Discussion}

We have presented evidence that circulating cholesterol, in isolation from other factors, stimulates the growth of human prostate tumors in vivo and, conversely, that cholesterol lowering slows tumor growth. Our conclusion that the effects we describe can be attributed to cholesterol is supported by the following evidence: 1) tumor growth closely corresponded to serum cholesterol level; 2) we used isocaloric diets, eliminating any potential effect of energy imbalance on tumor growth; 3) cholesterol lower- ing was accomplished with the drug ezetimibe (Zetia), which blocks both dietary and biliary cholesterol uptake by targeting NPC1L1, a gut transporter responsible for essentially all uptake of dietary cholesterol ${ }^{40-42}$; ezetimibe is believed at this time to be specific for NPC1LI; 4) the diet interventions did not affect testosterone levels (data not shown), consequently these results cannot be attributed to availability of androgen; 5) triglyceride levels were not altered by diet or decreased by ezetimibe treatment, and all liver function tests were normal; and 6) serum cholesterol levels correlated significantly with tumor cholesterol levels, apoptotic tumor cell number, tumor cell proliferation, and MVD.

The most dramatic effect elicited by the manipulation of circulating cholesterol levels was its impact on tumor angiogenesis. Our studies revealed that reducing cholesterol levels reduces the amount of tumor-associated blood and blood vessels, and increases vessel pericyte coverage (suggesting more stable vascular structure), while raising the tumor-associated levels of TSP-1, a potent inhibitor of angiogenesis. These results strongly suggest that a major biological effect of hypercholesterolemia on prostate tumors is increased angiogenesis. An important implication of these findings is the possibility that cholesterol reduction, which is routinely accomplished pharmacologically in humans, may reduce angiogenesis, ultimately leading to less aggressive tumors. These data are consistent with published data showing that long-term ( $>5 \mathrm{yrs}$ ) cholesterol lowering therapy, principally through the use of statin drugs, reduces the risk of advanced PCa. ${ }^{10,13-15}$ These data further suggest that an anti-angiogenic mechanism may account for significant chemopreventive effects against PCa observed in patients treated with statins for a prolonged period.

The increase in endothelial cell numbers as a consequence of hypercholesterolemia suggested that tumors might also exhibit an increase in other stromal cell components. We analyzed the tumors using a fibroblast-specific maker and determined that the hypercholesterolemic cohort (ie, fed the HFHC diet without ezetimibe) had a statistically significant greater number of fibroblasts than the other cohorts, although as a proportion of the total number of cells in the tumors, the fibroblasts were in general a very small cellular component (see Figure 4A). A role for stromal fibroblasts in promoting tumor progression has been described, including the ability of stromal fibroblasts to stimulate angiogenesis through increased levels of stromal cell-derived factor- $1 .{ }^{60,61}$ Cholesterol has been shown to promote proliferation and to regulate nitric oxide signaling in fibroblasts, ${ }^{62,63}$ but a specific role for cholesterol in activities that may mediate tumor fibroblast stimulation of tumor growth has not, to our knowledge, been described. Our current efforts suggest that hypercholesterolemia either increases the recruitment or in situ proliferation of stromal fibroblasts, where they perhaps contribute to the promotion of tumor growth.

In these experiments we demonstrate that ezetimibe blocks the accelerated tumor growth stimulated by the HFHC diet, and even reduces the more modest tumor growth in mice fed a LFNC diet. Ezetimibe works by 
blocking intestinal uptake of dietary cholesterol and bilederived cholesterol; thus, the drug is expected to reduce circulating cholesterol levels even when there is no cholesterol in the diet. ${ }^{41-44}$ Consistent with this expectation, we found that ezetimibe reduced serum cholesterol levels in mice with no cholesterol in their diet. Unlike statins, which block cholesterol synthesis at an early step in the mevalonate pathway, and thus suppress production of upstream intermediates (including isoprenoids), ezetimibe only blocks cholesterol-uptake and likely has no direct effect on other components of the pathway. Ezetimibe has been shown to have a modest effect on reducing triglyceride levels in humans, ${ }^{41,42}$ but in our studies in mice we found no reduction of serum triglyceride levels, suggesting that altered triglycerides did not contribute to ezetimibe's effects. Our studies also demonstrate that a combination of a LFNC diet and ezetimibe reduces tumor growth additively.

Although we do not know the precise mechanism by which lower serum cholesterol results in reduced tumor growth, our investigations demonstrate that TSP-1 levels are inversely correlated to the level of serum cholesterol. TSP-1 is a multifunctional $450-\mathrm{kDa}$ extracellular matrix glycoprotein and the first endogenous inhibitor of angiogenesis to be discovered. It is critical to the formation and progression of solid tumors including regulating proliferation, adhesion, migration, and angiogenesis. Our observation that hypercholesterolemia decreases expression of the angiogenesis inhibitor TSP-1 is consistent with prior reports that TSP-1 overexpression inhibits the growth of prostate tumor xenografts in mice. ${ }^{64} \mathrm{LNCaP}$ cells express both TSP-1 as well as its major receptor CD36, ${ }^{65}$ and TSP-1 has been shown to directly inhibit cell proliferation and stimulate apoptosis. ${ }^{65}$ Thus, it is possible that under hypercholesterolemic conditions the reduction in TSP-1 levels is a direct contributor to the increased angiogenesis, increased tumor cell proliferation, and the decreased apoptosis that we observed. The potential for cholesterol to regulate TSP-1, and, potentially, other angiogenesis inhibitors, is not known, although one study reported increased TSP-1 expression in the rabbit aorta under hypercholesterolemic conditions. ${ }^{66}$ Regulation of vascular mediators by circulating cholesterol appears to be a productive area for investigation. Interestingly, CD36 localizes to cholesterol-enriched lipid raft membranes, ${ }^{67}$ a subcellular compartment that, in tumors, sequesters higher levels of cholesterol under conditions of hypercholesterolemia. ${ }^{12}$ Whether increased cholesterol in the serum and/or in tumor cell membranes alters TSP-1 signals mediated by CD36 is not known.

The results of these experiments cannot easily be extrapolated to anticipate the effects of cholesterol lowering/raising in the context of human prostate cancer, due to limitations of the model we used. First, the xenograft site (subcutaneous flank) has a very different vascular bed than that which is present in the prostate. Because angiogenesis appears to be a major mediator of ezetimibe's effect on tumor growth, the physiological actions of the drug may differ in situations where the degree of vascularity and proximity to the circulatory system are different. Second, because the experiments described here involve xenografts generated from tumorigenic cells, our studies only address a role for cholesterol in tumor progression, not tumor initiation. However, our studies do suggest the possibility that chemopreventive intervention to inhibit PCa progression by lowering cholesterol pharmacologically, possibly in combination with diet, may be effective in humans.

\section{Acknowledgments}

We thank Diane Bielenberg, Jack Lawler, and the late Judah Folkman, for their advice and critical comments.

\section{References}

1. Freeman MR, Solomon KR: Cholesterol and prostate cancer. J Cell Biochem 2004, 91:54-69

2. Schaffner CP: Prostatic cholesterol metabolism: regulation and alteration. Prog Clin Biol Res 1981, 75A:279-324

3. Swyer Gl: The cholesterol content of normal and enlarged prostates. Cancer Res 1942, 2:372-375

4. Blais L, Desgagne A, LeLorier J: 3-Hydroxy-3-methylglutaryl coenzyme A reductase inhibitors and the risk of cancer: a nested casecontrol study. Arch Intern Med 2000, 160:2363-2368

5. Bravi F, Scotti L, Bosetti C, Talamini R, Negri E, Montella M, Franceschi S, La Vecchia C: Self-reported history of hypercholesterolaemia and gallstones and the risk of prostate cancer. Ann Oncol 2006, 17:1014-1017

6. Cook LS, Goldoft M, Schwartz SM, Weiss NS: Incidence of adenocarcinoma of the prostate in Asian immigrants to the United States and their descendants. J Urol 1999, 161:152-155

7. Graaf MR, Beiderbeck AB, Egberts AC, Richel DJ, Guchelaar HJ: The risk of cancer in users of statins. J Clin Oncol 2004, 22:2388-2394

8. Pedersen TR, Wilhelmsen L, Faergeman O, Strandberg TE, Thorgeirsson G, Troedsson L, Kristianson J, Berg K, Cook TJ, Haghfelt T, Kjekshus J, Miettinen T, Olsson AG, Pyorala K, Wedel H: Follow-up study of patients randomized in the Scandinavian simvastatin survival study (4S) of cholesterol lowering. Am J Cardiol 2000, 86:257-262

9. Platz EA, Leitzmann MF, Visvanathan K, Stampfer MJ, Willett WC Rimm EB, Giovannucci E: Cholesterol-lowering drugs including stains and the risk of prostate cancer in a large prospective cohort study. AACR Meeting Abstracts 2005, 2005:1034-a-

10. Platz EA, Leitzmann MF, Visvanathan K, Rimm EB, Stampfer MJ, Willett WC, Giovannucci E: Statin drugs and risk of advanced prostate cancer. J Natl Cancer Inst 2006, 98:1819-1825

11. Pu YS, Chiang HS, Lin CC, Huang CY, Huang KH, Chen J: Changing trends of prostate cancer in Asia. Aging Male 2004, 7:120-132

12. Zhuang L, Kim J, Adam RM, Solomon KR, Freeman MR: Cholesterol targeting alters lipid raft composition and cell survival in prostate cancer cells and xenografts. J Clin Invest 2005, 115:959-968

13. Jacobs EJ, Rodriguez C, Bain EB, Wang Y, Thun MJ, Calle EE: Cholesterol-lowering drugs and advanced prostate cancer incidence in a large U.S. cohort. Cancer Epidemiol Biomarkers Prev 2007, 16:2213-2217

14. Murtola TJ, Tammela TL, Lahtela J, Auvinen A: Cholesterol-lowering drugs and prostate cancer risk: a population-based case-control study. Cancer Epidemiol Biomarkers Prev 2007, 16:2226-2232

15. Flick ED, Habel LA, Chan KA, Van Den Eeden SK, Quinn VP, Haque R, Orav EJ, Seeger JD, Sadler MC, Quesenberry CP, Jr., Sternfeld B, Jacobsen SJ, Whitmer RA, Caan BJ: Statin use and risk of prostate cancer in the California Men's Health Study Cohort. Cancer Epidemiol Biomarkers Prev 2007, 16:2218-2225

16. Kolonel LN, Nomura AM, Cooney RV: Dietary fat and prostate cancer current status. J Natl Cancer Inst 1999, 91:414-428

17. Michaud DS, Augustsson K, Rimm EB, Stampfer MJ, Willet WC, Giovannucci E: A prospective study on intake of animal products and risk of prostate cancer. Cancer Causes Control 2001, 12:557-567

18. Wu K, Hu FB, Willett WC, Giovannucci E: Dietary patterns and risk of prostate cancer in U.S. men. Cancer Epidemiol Biomarkers Prev 2006, 15:167-171

19. Walker M, Aronson KJ, King W, Wilson JW, Fan W, Heaton JP, 
MacNeily A, Nickel JC, Morales A: Dietary patterns and risk of prostate cancer in Ontario. Canada, Int J Cancer 2005, 116:592-598

20. Dale KM, Coleman CI, Henyan NN, Kluger J, White CM: Statins and cancer risk: a meta-analysis. JAMA 2006, 295:74-80

21. Browning DR, Martin RM: Statins and risk of cancer: a systematic review and metaanalysis. Int J Cancer 2007, 120:833-843

22. Baigent C, Keech A, Kearney PM, Blackwell L, Buck G, Pollicino C, Kirby A, Sourjina T, Peto R, Collins R, Simes R: Efficacy and safety of cholesterol-lowering treatment: prospective meta-analysis of data from 90,056 participants in 14 randomised trials of statins. Lancet 2005, 366:1267-1278

23. Duncan RE, EI-Sohemy A, Archer MC: Statins and the risk of cancer. JAMA 2006, 295:2720; author reply 2721-2722

24. Freeman MR, Solomon KR, Moyad M: Statins and the risk of cancer. JAMA 2006, 295:2720-2721; author reply 2721-2722

25. Salinas CA, Agalliu I, Stanford JL, Lin DW: Statins and the risk of cancer. JAMA 2006, 295:2721; author reply 2721-2722

26. Boucher K, Siegel CS, Sharma P, Hauschka PV, Solomon KR: HMGCoA reductase inhibitors induce apoptosis in pericytes. Microvasc Res 2006, 71:91-102

27. Bassa BV, Roh DD, Vaziri ND, Kirschenbaum MA, Kamanna VS: Effect of inhibition of cholesterol synthetic pathway on the activation of Ras and MAP kinase in mesangial cells. Biochim Biophys Acta 1999, 1449:137-149

28. lishi H, Tatsuta M, Baba M, Yano H, Sakai N, Uehara H, Nakaizumi A: ras p21 Isoprenylation inhibition induces flat colon tumors in Wistar rats. Dis Colon Rectum 2000, 43:70-75

29. Macaulay RJ, Wang W, Dimitroulakos J, Becker LE, Yeger H: Lovastatin-induced apoptosis of human medulloblastoma cell lines in vitro. J Neurooncol 1999, 42:1-11

30. Matar P, Rozados VR, Roggero EA, Scharovsky OG: Lovastatin inhibits tumor growth and metastasis development of a rat fibrosarcoma. Cancer Biother Radiopharm 1998, 13:387-393

31. Park HJ, Kong D, Iruela-Arispe L, Begley U, Tang D, Galper JB: 3-hydroxy-3-methylglutaryl coenzyme A reductase inhibitors interfere with angiogenesis by inhibiting the geranylgeranylation of RhoA. Circ Res 2002, 91:143-150

32. Resh MD: Regulation of cellular signalling by fatty acid acylation and prenylation of signal transduction proteins. Cell Signal 1996, 8:403-412

33. Sebti SM, Tkalcevic GT, Jani JP: Lovastatin, a cholesterol biosynthesis inhibitor, inhibits the growth of human H-ras oncogene transformed cells in nude mice. Cancer Commun 1991, 3:141-147

34. Unlu S, Clunn G, Schachter M, Demoliou-Mason C, Hughes AD: Action of an HMG CoA reductase inhibitor, lovastatin, on apoptosis of untransformed and ts-SV40 transformed human smooth muscle cells derived from saphenous vein. J Cardiovasc Pharmacol 2001, 38:161-173

35. Werner N, Nickenig G, Laufs U: Pleiotropic effects of HMG-CoA reductase inhibitors. Basic Res Cardiol 2002, 97:105-116

36. Desager JP, Horsmans Y: Clinical pharmacokinetics of 3-hydroxy-3methylglutaryl-coenzyme A reductase inhibitors. Clin Pharmacokinet 1996, 31:348-371

37. Sirtori CR: Tissue selectivity of hydroxymethylglutaryl coenzyme A (HMG CoA) reductase inhibitors. Pharmacol Ther 1993, 60:431-459

38. Solomon KR, Freeman MR: Do the cholesterol-lowering properties of statins affect cancer risk? Trends Endo Met 2007, 19:113-121

39. Zhuang L, Lin J, Lu ML, Solomon KR, Freeman MR: Cholesterol-rich lipid rafts mediate akt-regulated survival in prostate cancer cells. Cancer Res 2002, 62:2227-2231

40. Davis HR, Veltri EP: Zetia: inhibition of Niemann-Pick C1 Like 1 (NPC1L1) to reduce intestinal cholesterol absorption and treat hyperlipidemia. J Atheroscler Thromb 2007, 14:99-108

41. Jurado J, Seip R, Thompson PD: Effectiveness of ezetimibe in clinical practice. Am J Cardiol 2004, 93:641-643

42. Knopp RH, Gitter H, Truitt T, Bays H, Manion CV, Lipka LJ, LeBeaut AP, Suresh R, Yang B, Veltri EP: Effects of ezetimibe, a new cholesterol absorption inhibitor, on plasma lipids in patients with primary hypercholesterolemia. Eur Heart J 2003, 24:729-741

43. Altmann SW, Davis HR, Jr., Zhu LJ, Yao X, Hoos LM, Tetzloff G, lyer SP, Maguire M, Golovko A, Zeng M, Wang L, Murgolo N, Graziano MP: Niemann-Pick C1 Like 1 protein is critical for intestinal cholesterol absorption. Science 2004, 303:1201-1204

44. Davis HR, Jr., Zhu LJ, Hoos LM, Tetzloff G, Maguire M, Liu J, Yao X, lyer SP, Lam MH, Lund EG, Detmers PA, Graziano MP, Altmann SW:
Niemann-Pick C1 Like 1 (NPC1L1) is the intestinal phytosterol and cholesterol transporter and a key modulator of whole-body cholesterol homeostasis. J Biol Chem 2004, 279:33586-33592

45. Wu X, Senechal K, Neshat MS, Whang YE, Sawyers CL: The PTEN/ MMAC1 tumor suppressor phosphatase functions as a negative regulator of the phosphoinositide 3-kinase/Akt pathway. Proc Natl Acad Sci USA 1998, 95:15587-15591

46. Bielenberg DR, Hida Y, Shimizu A, Kaipainen A, Kreuter M, Kim CC, Klagsbrun M: Semaphorin 3F, a chemorepulsant for endothelial cells, induces a poorly vascularized, encapsulated, nonmetastatic tumor phenotype. J Clin Invest 2004, 114:1260-1271

47. Solomon KR, Danciu TE, Adolphson LD, Hecht LE, Hauschka PV Caveolin-enriched membrane signaling complexes in human and murine osteoblasts. J Bone Miner Res 2000, 15:2380-2390

48. Solomon KR, Mallory MA, Finberg RW: Determination of the non-ionic detergent insolubility and phosphoprotein associations of glycosylphosphatidylinositol-anchored proteins expressed on T cells. Biochem J 1998, 334:325-333

49. Boyle MD, Ohanian SH: Evidence for the influence of the initial complement components on the assembly and activity of the membrane attack complex. J Immunol 1980, 124:2824-2827

50. Gee AP, Boyle MD, Borsos T: Distinction between C8-mediated and C8/C9-mediated hemolysis on the basis of independent $86 \mathrm{Rb}$ and hemoglobin release. J Immunol 1980, 124:1905-1910

51. Laird NM, Ware JH: Random-effects models for longitudinal data. Biometrics 1982, 38:963-974

52. Akaike H: Likelihood of a model and information criteria. J Econometrics 1981, 16:3-14

53. Montgomery D: Design and analysis of experiments. New York, John Wiley \& Sons, 2001, pp. 170-217

54. Paigen B: Genetics of responsiveness to high-fat and high-cholesterol diets in the mouse. Am J Clin Nutr 1995, 62:458S-462S

55. Lichtman AH, Clinton SK, liyama K, Connelly PW, Libby P, Cybulsky MI: Hyperlipidemia and atherosclerotic lesion development in LDL receptor-deficient mice fed defined semipurified diets with and without cholate. Arterioscler Thromb Vasc Biol 1999, 19:1938-1944

56. Gratton JP, Bernatchez P, Sessa WC: Caveolae and caveolins in the cardiovascular system. Circ Res 2004, 94:1408-1417

57. Lu ML, Schneider MC, Zheng Y, Zhang X, Richie JP: Caveolin- 1 interacts with androgen receptor. A positive modulator of androgen receptor mediated transactivation, J Biol Chem 2001, 276:13442-13451

58. Bergers G, Song S: The role of pericytes in blood-vessel formation and maintenance. Neuro Oncol 2005, 7:452-464

59. Niu Q, Perruzzi C, Voskas D, Lawler J, Dumont DJ, Benjamin LE: Inhibition of Tie-2 signaling induces endothelial cell apoptosis, decreases Akt signaling, and induces endothelial cell expression of the endogenous anti-angiogenic molecule, thrombospondin-1. Cancer Biol Ther 2004, 3:402-405

60. Orimo A, Gupta PB, Sgroi DC, Arenzana-Seisdedos F, Delaunay T, Naeem R, Carey VJ, Richardson AL, Weinberg RA: Stromal fibroblasts present in invasive human breast carcinomas promote tumor growth and angiogenesis through elevated SDF-1/CXCL12 secretion. Cell 2005, 121:335-348

61. Orimo A, Weinberg RA: Stromal fibroblasts in cancer: a novel tumorpromoting cell type. Cell Cycle 2006, 5:1597-1601

62. Chen BY, Wei JG, Wang YC, Yu J, Qian JX, Chen YM, Xu J: Effects of cholesterol on proliferation and functional protein expression in rabbit bile duct fibroblasts. World J Gastroenterol 2004, 10:889-893

63. Miersch S, Espey MG, Chaube R, Akarca A, Tweten R, Ananvoranich S, Mutus B: Plasma membrane cholesterol content affects nitric oxide diffusion dynamics and signaling. J Biol Chem 2008, 283:18513-18521

64. Jin RJ, Kwak C, Lee SG, Lee CH, Soo CG, Park MS, Lee E, Lee SE: The application of an anti-angiogenic gene (thrombospondin-1) in the treatment of human prostate cancer xenografts. Cancer Gene Ther 2000, 7:1537-1542

65. Bastian M, Steiner M, Schuff-Werner P: Expression of thrombospondin-1 in prostate-derived cell lines. Int J Mol Med 2005, 15:49-56

66. Roth JJ, Gahtan V, Brown JL, Gerhard C, Swami VK, Rothman VL, Tulenko TN, Tuszynski GP: Thrombospondin-1 is elevated with both intimal hyperplasia and hypercholesterolemia. J Surg Res 1998, 74:11-16

67. Febbraio M, Hajjar DP, Silverstein RL: CD36: a class B scavenger receptor involved in angiogenesis, atherosclerosis, inflammation, and lipid metabolism. J Clin Invest 2001, 108:785-791 